\title{
THE DIMENSION SPECTRUM OF PROJECTED MEASURES ON RIEMANN MANIFOLDS
}

\author{
Risto Hovila \\ University of Jyväskylä, Department of Mathematics and Statistics \\ P.O. Box 35, FI-40014 University of Jyväskylä, Finland; and \\ University of Helsinki, Department of Mathematics and Statistics \\ P.O. Box 68, FI-00014 University of Helsinki, Finland; risto.hovila@helsinki.fi
}

\begin{abstract}
In this work we first generalize the projection results concerning the dimension spectrum of projected measures on $\mathbf{R}^{n}$ to parametrized families of transversal mappings between smooth manifolds and measures on them. The projection theorems for the lower $q$-dimension were first considered in [FO] and [HK]. Theorems for the upper $q$-dimension were first considered in [FO] and [JJ]. After proving the generalized results, we compute for $1<q \leq 2$ the lower and the upper $q$-dimensions of the natural projection of a probability measure which is invariant under the geodesic flow on the unit tangent bundle of a two-dimensional Riemann manifold.
\end{abstract}

\section{Introduction}

The dimensional properties of projections of sets and measures have been studied for decades. This study was initiated by Marstrand in 1954. In [Mar] he proved a wellknown theorem according to which the Hausdorff dimension, $\operatorname{dim}_{\mathrm{H}}$, of a planar set is preserved under typical orthogonal projections. In 1968 Kaufman $[\mathrm{K}]$ reproved the same result using potential theoretic methods and in 1975 Mattila [Mat1] generalized it to higher dimensions. For measures the analogous principle was discovered by Kaufman [K], Mattila [Mat2], Hu and Taylor [HT] and Falconer and Mattila [FM]. It can be stated in the following form: if $\mu$ is a compactly supported Radon measure on $\mathbf{R}^{n}$, then for almost all $V \in G(n, m)$

$$
\operatorname{dim}_{\mathrm{H}} P_{V *} \mu=\operatorname{dim}_{\mathrm{H}} \mu \text { provided that } \operatorname{dim}_{\mathrm{H}} \mu \leq m .
$$

Here we mean almost all with respect to the natural orthogonally invariant probability measure on $G(n, m)$. On the other hand, if $\operatorname{dim}_{\mathrm{H}} \mu>m$, then for almost all $V \in G(n, m)$

$$
\left.P_{V *} \mu \ll \mathscr{H}^{m}\right|_{V}
$$

In addition, if the $m$-energy of $\mu$ is finite, then for almost all $V \in G(n, m)$

$$
\left.P_{V *} \mu \ll \mathscr{H}^{m}\right|_{V} \text { with the Radon-Nikodym derivative in } L^{2}\left(V,\left.\mathscr{H}^{m}\right|_{V}\right) \text {. }
$$

Above $G(n, m)$ denotes the Grassmann manifold of all $m$-dimensional linear subspaces of $\mathbf{R}^{n}, P_{V}: \mathbf{R}^{n} \rightarrow V$ is the orthogonal projection onto $V \in G(n, m)$ and $P_{V *} \mu$ is the image measure of $\mu$ under the projection $P_{V}$. In addition, by $\mu \ll \nu$ we denote the absolute continuity of a measure $\mu$ with respect to a measure $\nu$, and $\left.\mu\right|_{A}$ is the restriction of a measure $\mu$ to a set $A$.

doi:10.5186/aasfm.2010.3537

2000 Mathematics Subject Classification: Primary 58C35, 53D25, 28A80, 37A05, 37D40.

Key words: Projection, dimension spectrum, measure, geodesic flow.

I acknowledge the support of the Centre of Excellence in Analysis and Dynamics Research funded by the Academy of Finland. 
The geometry of the packing dimension, $\operatorname{dim}_{\mathrm{p}}$, is less regular then that of the Hausdorff dimension. Falconer and Howroyd [FH] proved the following theorem for the packing dimension of projected measures: if $\mu$ is a finite Borel measure on $\mathbf{R}^{n}$, then

$$
\operatorname{dim}_{\mathrm{p}} P_{V_{*}} \mu=\operatorname{dim}_{m} \mu
$$

for almost all $V \in G(n, m)$, where $\operatorname{dim}_{m}$ is a packing type dimension defined by using a certain $m$-dimensional kernel. It can happen that $\operatorname{dim}_{m} \mu<\operatorname{dim}_{\mathrm{p}} \mu$, which means that the dimension can decrease under typical projections, but still the packing dimension is the same for almost all projections.

Hunt and Kaloshin [HK] showed that the lower $q$-dimension, $\underline{D}_{q}$, behaves similar to the Hausdorff dimension under projections, provided that $1<q \leq 2$ (for the definition of $q$-dimensions, see section 2). They proved the following theorem using potential theoretic methods: Let $1<q \leq 2$ and let $\mu$ be a compactly supported Borel probability measure on $\mathbf{R}^{n}$. Then

$$
\underline{D}_{q}\left(P_{V *} \mu\right)=\min \left\{m, \underline{D}_{q}(\mu)\right\}
$$

for almost all $V \in G(n, m)$. Falconer and O'Neil [FO] reproved their theorem by studying certain appropriately defined convolution kernels. By these methods they also proved the following projection theorem for the upper $q$-dimension, which shows that the behaviour of the upper $q$-dimension is similar to that of the packing dimension: Let $\mu$ be a compactly supported Borel probability measure on $\mathbf{R}^{n}$. Then

$$
\bar{D}_{q}\left(P_{V *} \mu\right)=\bar{D}_{q}^{m}(\mu)
$$

for almost all $V \in G(n, m)$, where $\bar{D}_{q}^{m}(\mu)$ is a dimension defined by using the $m$ dimensional kernel mentioned above. As in the case of the packing dimension, it can happen that $\bar{D}_{q}^{m}(\mu)<\bar{D}_{q}(\mu)$. In [JJ] E. Järvenpää and M. Järvenpää also considered the upper $q$-dimension using potential theoretic methods and they presented an alternative proof for the above theorem.

All the results mentioned above are "almost all"-results, which give no information about any specific projection. However, Ledrappier and Lindenstrauss discovered that similar methods work for one specific projection. In [LL] they studied measures on the unit tangent bundle $S M$ of a compact Riemann surface $M$. They showed that if $\mu$ is a Radon probability measure on $S M$ and $\mu$ is invariant under the geodesic flow, then

$$
\operatorname{dim}_{\mathrm{H}} \Pi_{*} \mu=\operatorname{dim}_{\mathrm{H}} \mu \text { if } \operatorname{dim}_{\mathrm{H}} \mu \leq 2 \text {, and }\left.\Pi_{*} \mu \ll \mathscr{H}^{2}\right|_{M} \text { if } \operatorname{dim}_{\mathrm{H}} \mu>2 \text {. }
$$

Here $\Pi: S M \rightarrow M$ is the natural projection. E. Järvenpää, M. Järvenpää and Leikas [JJLe] reproved the above theorem using the generalized projection formalism introduced by Peres and Schlag in [PS]. Their proof also explains why the above theorem fails in higher dimensions. Leikas [Le] proved an analogous theorem for the packing dimension using similar methods.

In this paper we consider the question of how the dimension spectrum of an invariant measure behaves under the natural projection. In order to do this, we first generalize the results from [FO], [HK] and [JJ] to parametrized families of transversal mappings between smooth manifolds and measures on them (Theorem 3.8). We use similar methods to those of Falconer and O'Neil. However, their methods do not directly work in our setting, but circumventing some technical problems eventually leads to a similar proof. After this we compute the dimension spectrum of $\Pi_{*} \mu$ in 
a similar setting as in [LL] (Theorem 4.2). Altough the result for the $q$-dimension is similar to those concerning the Hausdorff dimension and the packing dimension, we have to use different methods for the proof because the $q$-dimensions of slices of a measure do not behave as well as the Hausdorff or the packing dimension (see Remark 4.4). Finally, we give an example of a locally invariant measure, whose upper $q$-dimension decreases under the projection even in the two-dimensional case.

\section{Preliminaries and definitions}

In the following definitions $(X, d)$ is a metric space. We denote by $B(x, r)$ the open ball with center at $x \in X$ and radius $r>0$.

Definition 2.1. Let $\mu$ be a Borel probability measure on $X$ with compact support. For every $q \neq 1$ we define the lower and upper $q$-dimensions by

$$
\underline{D}_{q}(\mu)=\liminf _{r \rightarrow 0} \frac{\log \int \mu(B(x, r))^{q-1} d \mu(x)}{(q-1) \log r}
$$

and

$$
\bar{D}_{q}(\mu)=\limsup _{r \rightarrow 0} \frac{\log \int \mu(B(x, r))^{q-1} d \mu(x)}{(q-1) \log r} .
$$

If $q>1$ and if $X=\mathbf{R}^{n}$, these definitions are equivalent to

$$
\underline{D}_{q}(\mu)=\liminf _{r \rightarrow 0} \frac{\log \sum_{C \in \mathscr{C}_{r}} \mu(C)^{q}}{(q-1) \log r}
$$

and

$$
\bar{D}_{q}(\mu)=\limsup _{r \rightarrow 0} \frac{\log \sum_{C \in \mathscr{C}_{r}} \mu(C)^{q}}{(q-1) \log r}
$$

where the sums are over all $r$-mesh cubes in $\mathbf{R}^{n}$, see $[\mathrm{FO}]$ and $[\mathrm{HP}]$. In this paper we consider the $q$-dimensions only for $q>1$.

As in [FO], for every $k \in \mathbf{N}$ we define a new dimension of a measure, which is defined by means of convolving the measure with a certain $k$-dimensional kernel.

Definition 2.2. Let $\mu$ be a Borel probability measure on $X$ with compact support. For every $x \in X, r>0$ and $k \in \mathbf{N}$ we define

$$
F_{\mu}^{k}(x, r)=\int_{X} \min \left\{1, r^{k} d(x, y)^{-k}\right\} d \mu(y)=k r^{k} \int_{r}^{\infty} \frac{\mu(B(x, h))}{h^{k+1}} d h,
$$

where the last inequality follows from Fubini's theorem. Furthermore, for $q \neq 1$ we define

$$
\underline{D}_{q}^{k}(\mu)=\liminf _{r \rightarrow 0} \frac{\log \int F_{\mu}^{k}(x, r)^{q-1} d \mu(x)}{(q-1) \log r}
$$

and

$$
\bar{D}_{q}^{k}(\mu)=\limsup _{r \rightarrow 0} \frac{\log \int F_{\mu}^{k}(x, r)^{q-1} d \mu(x)}{(q-1) \log r} .
$$

The following technical lemma from $[\mathrm{FO}]$ is needed later on. 
Lemma 2.3. Let $p \geq 0$. There exists $c>0$ depending only on $n$ and $p$ such that for any compactly supported finite Borel regular measure $\mu$ on $\mathbf{R}^{n}$ and for all $0<r \leq h$

$$
\int \mu(B(x, h))^{p} d \mu(x) \leq c\left(\frac{h}{r}\right)^{n p} \int \mu(B(x, r))^{p} d \mu(x) .
$$

Proof. See Lemma 2.7 from [FO].

The following theorem concerning the dimension spectrum of projected measures is also from $[\mathrm{FO}]$.

Theorem 2.4. Let $\mu$ be a compactly supported Borel regular probability measure on $\mathbf{R}^{n}$ and let $1 \leq m \leq n$. Then for all $q>1$ and all $V \in G(n, m)$

$$
\underline{D}_{q}\left(\mu_{V}\right) \leq \underline{D}_{q}^{m}(\mu)=\min \left\{m, \underline{D}_{q}(\mu)\right\} \text { and } \bar{D}_{q}\left(\mu_{V}\right) \leq \bar{D}_{q}^{m}(\mu) \text {. }
$$

Moreover, for all $1<q \leq 2$ and for $\gamma_{n, m}$-almost all $V \in G(n, m)$

$$
\underline{D}_{q}\left(\mu_{V}\right)=\underline{D}_{q}^{m}(\mu)=\min \left\{m, \underline{D}_{q}(\mu)\right\} \text { and } \bar{D}_{q}\left(\mu_{V}\right)=\bar{D}_{q}^{m}(\mu) .
$$

Here $\mu_{V}=P_{V *} \mu$ is the image measure under the orthogonal projection onto an $m$-plane $V$.

Proof. See Theorem 3.9 from [FO].

\section{The dimension spectrum and transversal mappings between manifolds}

In this section we generalize Theorem 2.4 to families of transversal mappings. First we state two propositions from [FO] for later use.

Proposition 3.1. Let $\left(N, d_{N}\right)$ be a smooth $n$-dimensional Riemann manifold equipped with the distance function $d_{N}$ induced by the Riemann metric. Let $1 \leq$ $m \leq n, p>0, \epsilon>0$ and $R>1$. Then there exists a constant $c>0$ such that for any compactly supported Borel regular probability measure $\mu$ on $N$ with spt $\mu \subset B(y, R)$ for some $y \in N$, and for any $0<r \leq 1$

$$
\int_{N} F_{\mu}^{m}(x, r)^{p} d \mu(x) \leq c r^{m p-\epsilon} \int_{r}^{\infty} h^{-m p-1} \int_{N} \mu(B(x, h))^{p} d \mu(x) d h .
$$

Here spt $\mu$ is the support of the measure $\mu$.

Proof. See Proposition 2.5 in [FO]. The same proof works also in this more general setting, since one only needs a lemma for non-decreasing functions $f:[0, \infty) \rightarrow$ $[0, \infty)$ such that $f(h)$ is constant for all large enough $h$. The function for which the lemma is applied is $h \mapsto \mu(B(x, h))$, where $x \in \operatorname{spt} \mu$ is fixed.

The next proposition from [FO] states that the lower $q$-dimension of a measure $\mu$ is the same as the lower dimension defined using the kernel $F_{\mu}^{m}(x, r)$.

Proposition 3.2. Let $\left(N, d_{N}\right)$ be a smooth $n$-dimensional Riemann manifold equipped with the distance function $d_{N}$ induced by the Riemann metric and let $\mu$ be a compactly supported Borel regular probability measure on $N$. Then for $1 \leq m \leq n$ and $q>1$

$$
\underline{D}_{q}^{m}(\mu)=\min \left\{m, \underline{D}_{q}(\mu)\right\}
$$


Proof. See [FO, Proposition 3.8]. Again the same proof works also in this more general setting, since it is based on [FO, Proposition 2.5], which was above noticed to hold also in our case.

Next we introduce the setting we are going to work with. Let $\left(L, d_{L}\right)$ be a smooth, $l$-dimensional Riemann manifold equipped with the distance function $d_{L}$ induced by the Riemann metric, let $\left(N, d_{N}\right)$ be a smooth $n$-dimensional Riemann manifold and let $\left(M, d_{M}\right)$ be a smooth $m$-dimensional Riemann manifold. We suppose that $l, n \geq m$. Let $P: L \times N \rightarrow M, P(\lambda, x)=P_{\lambda}(x)$ be a continuous mapping such that for all $j \in\{0,1,2\}$ there exists a constant $C_{j}$ such that

$$
\left\|D_{\lambda}^{j} P(\lambda, x)\right\| \leq C_{j}
$$

for all $(\lambda, x) \in L \times N$. The basic assumptions we need are the following:

(1) There exist collections $\mathscr{A}_{L}=\{(V, \psi)\}$ and $\mathscr{A}_{M}=\{(U, \varphi)\}$ of charts on $L$ and $M$, respectively, such that $\mathscr{A}_{L}$ is a cover of $L, \mathscr{A}_{M}$ is a cover of $M$ and the Lipschitz constants of the mappings $\varphi, \varphi^{-1}, \psi$ and $\psi^{-1}$ are bounded from above by a constant $K \geq 1$, which may depend on the mapping.

(2) Define

$$
\begin{aligned}
X=\left\{(\lambda, x, y) \in L \times N^{2}:\right. & x \neq y, \text { there exists } \\
& \left.(U, \varphi) \in \mathscr{A}_{M} \text { such that } P_{\lambda}(x), P_{\lambda}(y) \in U\right\} .
\end{aligned}
$$

We assume that the mapping $T: X \rightarrow \mathbf{R}^{m}$,

$$
T_{x, y}(\lambda)=\frac{\varphi \circ P_{\lambda}(x)-\varphi \circ P_{\lambda}(y)}{d_{N}(x, y)}
$$

is transversal, i.e., there exists a constant $C_{T}>0$ (which might depend on the chart $(U, \varphi)$ used in the definition of $T)$ such that the property

$$
\left|T_{x, y}(\lambda)\right| \leq C_{T}
$$

implies that

$$
\operatorname{det}\left(D T_{x, y}(\lambda)\left(D T_{x, y}(\lambda)\right)^{T}\right) \geq C_{T}^{2}
$$

Here $D$ is the derivative with respect to $\lambda$ and $A^{T}$ stands for the transpose of a matrix $A$. We refer to this property by saying that $P$ is transversal.

Moreover, we assume that for any chart $(V, \psi) \in \mathscr{A}_{L}$ there exists a constant $C_{L}$ such that

$$
\left|\partial_{\lambda_{j}} \partial_{\lambda_{k}} T_{x, y}(\lambda)_{i}\right| \leq C_{L}
$$

for all $j, k \in\{1, \ldots, l\}, i \in\{1, \ldots, m\}$, and for all $\lambda \in V, x, y \in N$ such that $(\lambda, x, y) \in X$.

From now on we will assume that the manifolds $\left(L, d_{L}\right),\left(N, d_{N}\right)$ and $\left(M, d_{M}\right)$, and the mapping $P: L \times N \rightarrow M$ satisfy the above assumptions.

Before stating the main result of this section, we will prove results which will eventually lead to a proof of our main theorem. In the next proposition the transversality condition plays a crucial role. The proposition is very similar to [JJN, Proposition 3.1]. However, our result is a bit weaker due to our weaker assumptions. 
Proposition 3.3. Let $\left(L, d_{L}\right),\left(N, d_{N}\right),\left(M, d_{M}\right)$ and $P: L \times N \rightarrow M$ be as above and let $\widetilde{N} \subset N$ be compact. Then for all $\lambda_{0} \in L$ there exist constants $C_{\lambda_{0}}>0$ and $r_{\lambda_{0}}>0$ such that

$$
\mathscr{H}^{l}\left\{\lambda \in B\left(\lambda_{0}, r_{\lambda_{0}}\right): d_{M}\left(P_{\lambda}(x), P_{\lambda}(y)\right) \leq r\right\} \leq C_{\lambda_{0}} \min \left\{1, r^{m} d_{N}(x, y)^{-m}\right\}
$$

for all $0<r \leq r_{\lambda_{0}}$ and for all distinct $x, y \in \tilde{N}$.

Proof. Fix $\lambda_{0} \in L$. Since $\widetilde{N}$ is compact, the set $P_{\lambda_{0}}(\widetilde{N})$ is also compact. Thus we can choose $r_{1}>0$ such that the closed $r_{1}$-neighbourhood of the set $P_{\lambda_{0}}(\widetilde{N})$, denoted by $\bar{B}\left(P_{\lambda_{0}}(\widetilde{N}), r_{1}\right)$, is compact. This follows from the fact that the set $P_{\lambda_{0}}(\widetilde{N})$ can be covered by finitely many precompact coordinate neighbourhoods. Moreover, we can assume that $B\left(\lambda_{0}, r_{1}\right) \subset V$ for some $V \in \mathscr{A}_{L}$. By Lebesgue's number lemma there exists $r_{2}<r_{1} / 2$ such that for some finite subcollection $\left\{\left(U_{i}, \varphi_{i}\right)\right\}_{i=1}^{k} \subset \mathscr{A}_{M}$ it holds that for any $p \in \bar{B}\left(P_{\lambda_{0}}(\widetilde{N}), r_{2}\right)$

$$
B\left(p, r_{2}\right) \subset U_{i} \quad \text { for some } i .
$$

Moreover, $B\left(\lambda, r_{2}\right) \subset B\left(\lambda_{0}, r_{1}\right) \subset V$ for all $\lambda \in B\left(\lambda_{0}, r_{2}\right)$. The proof itself is the same as the proof of [JJN, Proposition 3.1]. We only have to replace the manifold $L$ in their proof with the ball $B\left(\lambda_{0}, r_{2}\right)$, the manifold $M$ with the set $\bar{B}\left(P_{\lambda_{0}}(\widetilde{N}), r_{2}\right)$ and the constant $R$ with $r_{2}$. In this setting the assumptions of [JJN, Proposition 3.1] are satisfied. However, in this case the constants in the statement depend on $r_{2}$ and thus they depend on $\lambda_{0}$.

The next lemma is similar to Lemma 2.3. In the manifold $\left(M, d_{M}\right)$ we get a somewhat weaker result, but it is sufficient for our purposes.

Lemma 3.4. Let $\left(M, d_{M}\right)$ be as above, let $\mu$ be a compactly supported Borel regular propability measure on $M$ and let $p \geq 0$. Then there exist constants $R>0$ and $c_{1}>0$ such that if $0<h \leq R$, then for all $0<r \leq h$

$$
\int_{M} \mu(B(x, h))^{p} d \mu(x) \leq c_{1}\left(\frac{h}{r}\right)^{m p} \int_{M} \mu(B(x, r))^{p} d \mu(x) .
$$

Proof. By Lebesgue's number lemma, we find coordinate charts $\left(U_{i}, \varphi_{i}\right), i=$ $1, \ldots, k$, and a constant $R>0$ such that for all $x \in M$ there is an index $i \in\{1, \ldots, k\}$ for which spt $\mu \cap B(x, R) \subset U_{i}$. Since the Lipschitz constants of the mappings $\varphi_{i}$ and $\varphi_{i}^{-1}$ are uniformly bounded from above by a constant $K \geq 1$, we have that for any $x \in U_{i}$

$$
\varphi_{i}^{-1}\left(B\left(\varphi_{i}(x), K^{-1} r\right)\right) \subset U_{i} \cap B(x, r) \subset \varphi_{i}^{-1}\left(B\left(\varphi_{i}(x), K r\right)\right)
$$

for all $0<r \leq R$. Let $\mu_{i}=\left.\mu\right|_{U_{i}}$. By Lemma 2.3 the following holds: if $0<h \leq R$, then for all $0<r \leq h$

$$
\begin{aligned}
\int_{M} \mu(B(x, h))^{p} d \mu(x) & \leq \sum_{i=1}^{k} \int_{M} \mu_{i}(B(x, h))^{p} d \mu_{i}(x) \\
& \leq \sum_{i=1}^{k} \int_{M} \mu_{i}\left(\varphi_{i}^{-1}\left(B\left(\varphi_{i}(x), K h\right)\right)\right)^{p} d \mu_{i}(x) \\
& =\sum_{i=1}^{k} \int_{\mathbf{R}^{m}} \varphi_{i *} \mu_{i}(B(y, K h))^{p} d \varphi_{i *} \mu_{i}(y)
\end{aligned}
$$




$$
\begin{aligned}
& \leq \sum_{i=1}^{k} c\left(\frac{K^{2} h}{r}\right)^{m p} \int_{\mathbf{R}^{m}} \varphi_{i *} \mu_{i}\left(B\left(y, K^{-1} r\right)\right)^{p} d \varphi_{i *} \mu_{i}(y) \\
& \leq \sum_{i=1}^{k} c\left(\frac{K^{2} h}{r}\right)^{m p} \int_{M} \mu_{i}(B(x, r))^{p} d \mu_{i}(x) \\
& \leq c_{1}\left(\frac{h}{r}\right)^{m p} \int_{M} \mu(B(x, r))^{p} d \mu(x) .
\end{aligned}
$$

Thus the claim holds.

Combining Proposition 3.1 and Lemma 3.4 gives the following proposition.

Proposition 3.5. Let $\left(M, d_{M}\right)$ be as above, let $p \geq 0$ and $\epsilon>0$. Let $\mu$ be a compactly supported Borel regular probability measure on $M$. Then for sufficienly small $r$

$$
\int_{M} \mu(B(x, r))^{p} d \mu(x) \leq \int_{M} F_{\mu}^{m}(x, r)^{p} d \mu(x) \leq r^{-\epsilon} \int_{M} \mu(B(x, r))^{p} d \mu(x) .
$$

In particular, for $q>1$

$$
D_{q}(\mu)=D_{q}^{m}(\mu)
$$

Here $D_{q}$ and $D_{q}^{m}$ denote either the upper or the lower q-dimensions.

Proof. The left-hand inequality is clear. By Proposition 3.1 we have for small $r$

$$
\begin{aligned}
& \int_{M} F_{\mu}^{m}(x, r)^{p} d \mu(x) \\
& \leq c r^{m p-\epsilon} \int_{r}^{R} h^{-m p-1} \int_{M} \mu(B(x, h))^{p} d \mu(x) d h+c r^{m p-\epsilon} \int_{R}^{\infty} h^{-m p-1} d h,
\end{aligned}
$$

where $R$ is as in Lemma 3.4. Combining this with Lemma 3.4 gives

$$
\begin{aligned}
& \int_{M} F_{\mu}^{m}(x, r)^{p} d \mu(x) \leq c_{2} r^{-\epsilon} \int_{r}^{R} h^{-1} \int_{M} \mu(B(x, r))^{p} d \mu(x) d h+c_{2} r^{m p-\epsilon} R^{-m p} \\
& \leq c_{2} r^{-\epsilon}(\log R-\log r) \int_{M} \mu(B(x, r))^{p} d \mu(x)+c_{3} r^{-\epsilon} \int_{M} \mu(B(x, r))^{p} d \mu(x),
\end{aligned}
$$

since

$$
\left(\frac{r}{R}\right)^{m p} \leq c_{1} \frac{\int_{M} \mu(B(x, r))^{p} d \mu(x)}{\int_{M} \mu(B(x, R))^{p} d \mu(x)}
$$

by Lemma 3.4. Redefining $\epsilon$ gives the claim. Note that the term $\int_{M} \mu(B(x, R))^{p} d \mu(x)$ is a constant since $R$ is a constant and it is included in $c_{3}$.

The second statement follows from the first one by using it for $p=q-1$, taking logarithms, dividing by $(q-1) \log r$ and using the definitions of $q$-dimensions.

Proposition 3.6. Let $\left(L, d_{L}\right),\left(N, d_{N}\right),\left(M, d_{M}\right)$ and $P: L \times N \rightarrow M$ be as above, let $\mu$ be a compactly supported Borel regular probability measure on $N$ and let $\mu_{\lambda}=P_{\lambda *} \mu$.

1) Let $q \geq 1$. Given $\epsilon>0$, for all $\lambda \in L$,

$$
r^{\epsilon} \int_{N} F_{\mu}^{m}(x, r)^{q-1} d \mu(x) \leq \int_{M} \mu_{\lambda}(B(y, r))^{q-1} d \mu_{\lambda}(y)
$$


for sufficiently small $r$.

2) Let $1 \leq q \leq 2$. Given $\epsilon>0$, for $\mathscr{H}^{l}$-almost all $\lambda \in L$,

$$
\int_{M} \mu_{\lambda}(B(y, r))^{q-1} d \mu_{\lambda}(y) \leq r^{-\epsilon} \int_{N} F_{\mu}^{m}(x, r)^{q-1} d \mu(x)
$$

for sufficiently small $r$.

Proof. 1) Let $\lambda \in L$. First we notice that since $P_{\lambda}$ is Lipschitz on spt $\mu$, there exists a constant $c \geq 1$ such that

$$
F_{\mu}^{m}(x, r) \leq c F_{\mu_{\lambda}}^{m}\left(P_{\lambda}(x), r\right)
$$

for all $x \in \operatorname{spt} \mu$. Thus by Proposition 3.5

$$
\int_{N} F_{\mu}^{m}(x, r)^{q-1} d \mu(x) \leq c \int_{M} F_{\mu_{\lambda}}^{m}(y, r)^{q-1} d \mu_{\lambda}(y) \leq c r^{-\epsilon} \int_{M} \mu_{\lambda}(B(y, r))^{q-1} d \mu_{\lambda}(y)
$$

for small $r$. Redefining $\epsilon$ gives the claim.

2) Fix $\lambda_{0} \in L$. Using Proposition 3.3 we obtain constants $C_{\lambda_{0}}$ and $r_{\lambda_{0}}$ such that

$$
\mathscr{H}^{l}\left\{\lambda \in B\left(\lambda_{0}, r_{\lambda_{0}}\right): d_{M}\left(P_{\lambda}(x), P_{\lambda}(y)\right) \leq r\right\} \leq C_{\lambda_{0}} \min \left\{1, r^{m} d_{N}(x, y)^{-m}\right\}
$$

for all $0<r \leq r_{\lambda_{0}}$ and for all distinct $x, y \in \operatorname{spt} \mu$. Hence by Fubini's theorem

$$
\begin{aligned}
& \int_{B\left(\lambda_{0}, r_{\lambda_{0}}\right)} \mu_{\lambda}\left(B\left(P_{\lambda}(x), r\right)\right) d \mathscr{H}^{l}(\lambda)=\int_{B\left(\lambda_{0}, r_{\lambda_{0}}\right)} \mu\left(P_{\lambda}^{-1}\left(B\left(P_{\lambda}(x), r\right)\right)\right) d \mathscr{H}^{l}(\lambda) \\
& =\int_{B\left(\lambda_{0}, r_{\lambda_{0}}\right)} \mu\left\{y \in N: d_{M}\left(P_{\lambda}(x), P_{\lambda}(y)\right) \leq r\right\} d \mathscr{H}^{l}(\lambda) \\
& =\int_{N} \mathscr{H}^{l}\left\{\lambda \in B\left(\lambda_{0}, r_{\lambda_{0}}\right): d_{M}\left(P_{\lambda}(x), P_{\lambda}(y)\right) \leq r\right\} d \mu(y) \leq C_{\lambda_{0}} F_{\mu}^{m}(x, r),
\end{aligned}
$$

whenever $0<r<r_{\lambda_{0}}$. Using Jensen's inequality for the concave function $x \mapsto x^{q-1}$ we have for all $x \in \operatorname{spt} \mu$

$$
\begin{aligned}
\int_{B\left(\lambda_{0}, r_{\lambda_{0}}\right)} \mu_{\lambda}\left(B\left(P_{\lambda}(x), r\right)\right)^{q-1} d \mathscr{H}^{l}(\lambda) & \leq \widetilde{C}\left(\int_{B\left(\lambda_{0}, r_{\lambda_{0}}\right)} \mu_{\lambda}\left(B\left(P_{\lambda}(x), r\right)\right) d \mathscr{H}^{l}(\lambda)\right)^{q-1} \\
& \leq C_{1} F_{\mu}^{m}(x, r)^{q-1}
\end{aligned}
$$

for some constant $C_{1}$ independent of $r$. The constant $\widetilde{C}$ comes from the fact that the measure $\left.\mathscr{H}^{l}\right|_{B\left(\lambda_{0}, r_{\lambda_{0}}\right)}$ is not necessarily a probability measure. Integrating both sides with respect to $\mu$ and using Fubini's theorem gives

$$
\int_{B\left(\lambda_{0}, r_{\lambda_{0}}\right)} \int_{M} \mu_{\lambda}(B(y, r))^{q-1} d \mu_{\lambda}(y) d \mathscr{H}^{l}(\lambda) \leq C_{1} \int_{N} F_{\mu}^{m}(x, r)^{q-1} d \mu(x)
$$

for all $0<r<r_{\lambda_{0}}$. From this we see that for all $\alpha>0$ and for all $0<r<r_{\lambda_{0}}$

$$
\mathscr{H}^{l}\left\{\lambda \in B\left(\lambda_{0}, r_{\lambda_{0}}\right): \int_{M} \mu_{\lambda}(B(y, r))^{q-1} d \mu_{\lambda}(y)>\alpha \int_{N} F_{\mu}^{m}(x, r)^{q-1} d \mu(x)\right\} \leq C_{1} \alpha^{-1} .
$$

Using this for $r=2^{-k}$ and $\alpha=2^{k \epsilon}$, where $k$ is large enough, the Borel-Cantelli lemma implies that for $\mathscr{H}^{l}$-almost all $\lambda \in B\left(\lambda_{0}, r_{\lambda_{0}}\right)$ we have

$$
\int_{M} \mu_{\lambda}\left(B\left(y, 2^{-k}\right)\right)^{q-1} d \mu_{\lambda}(y) \leq 2^{k \epsilon} \int_{N} F_{\mu}^{m}\left(x, 2^{-k}\right)^{q-1} d \mu(x)
$$


for all sufficiently large integers $k$. Extrapolating to other $r$ and redefining $\epsilon$ appropriately we have that for $\mathscr{H}^{l}$-almost all $\lambda \in B\left(\lambda_{0}, r_{\lambda_{0}}\right)$

$$
\int_{M} \mu_{\lambda}(B(y, r))^{q-1} d \mu_{\lambda}(y) \leq r^{-\epsilon} \int_{N} F_{\mu}^{m}(x, r)^{q-1} d \mu(x)
$$

for all sufficiently small $r$. The claim follows from the fact that the manifold $L$ can be covered with countably many balls $B\left(\lambda_{0}, r_{\lambda_{0}}\right)$, since we assume the manifold to be second countable and therefore it is Lindelöf.

Theorem 3.7. Let $\left(L, d_{L}\right),\left(N, d_{N}\right),\left(M, d_{M}\right)$ and $P: L \times N \rightarrow M$ be as above, and let $\mu$ be a compactly supported Borel regular probability measure on $N$. Then for $q>1$ and all $\lambda \in L$,

$$
\liminf _{r \rightarrow 0}\left(\frac{\log \int F_{\mu}^{m}(x, r)^{q-1} d \mu(x)}{(q-1) \log r}-\frac{\log \int \mu_{\lambda}(B(x, r))^{q-1} d \mu_{\lambda}(x)}{(q-1) \log r}\right) \geq 0 .
$$

Moreover, for $1<q \leq 2$ and $\mathscr{H}^{l}$-almost all $\lambda \in L$,

$$
\lim _{r \rightarrow 0}\left(\frac{\log \int F_{\mu}^{m}(x, r)^{q-1} d \mu(x)}{(q-1) \log r}-\frac{\log \int \mu_{\lambda}(B(x, r))^{q-1} d \mu_{\lambda}(x)}{(q-1) \log r}\right)=0 .
$$

Here $\mu_{\lambda}=P_{\lambda *} \mu$.

Proof. This follows directly from Proposition 3.6.

Theorem 3.7 and Proposition 3.2 imply our main theorem of this section.

Theorem 3.8. Let $\left(L, d_{L}\right),\left(N, d_{N}\right),\left(M, d_{M}\right)$ and $P: L \times N \rightarrow M$ be as above, and let $\mu$ be a compactly supported Borel regular probability measure on $N$.

1) For all $q>1$ and all $\lambda \in L$

$$
\underline{D}_{q}\left(\mu_{\lambda}\right) \leq \underline{D}_{q}^{m}(\mu)=\min \left\{m, \underline{D}_{q}(\mu)\right\} \text { and } \bar{D}_{q}\left(\mu_{\lambda}\right) \leq \bar{D}_{q}^{m}(\mu) \text {. }
$$

2) For all $1<q \leq 2$ and $\mathscr{H}^{l}$-almost all $\lambda \in L$

$$
\underline{D}_{q}\left(\mu_{\lambda}\right)=\underline{D}_{q}^{m}(\mu)=\min \left\{m, \underline{D}_{q}(\mu)\right\} \text { and } \bar{D}_{q}\left(\mu_{\lambda}\right)=\bar{D}_{q}^{m}(\mu) \text {. }
$$

Here $\mu_{\lambda}=P_{\lambda *} \mu$.

Proof. This follows from Theorem 3.7, Proposition 3.2 and the definitions of $q$-dimensions.

\section{The dimension spectrum of the projection of a measure invariant under the geodesic flow}

In this section we study the natural projection of a two-dimensional Riemann manifold. The behaviour of the Hausdorff dimension of a locally invariant probability measure on the unit tangent bundle of a Riemann surface under the natural projection was studied in [LL] and [JJLe]. For the packing dimension this behaviour was studied in [Le]. By those results we know that the Hausdorff dimension is preserved under the natural projection, but the packing dimension can decrease. Next we will prove analogous theorems for the dimension spectrum of the projected measure. As one could expect, the lower $q$-dimension is preserved under the natural projection, while the upper one can decrease. 
Definition 4.1. Let $S M$ be the unit tangent bundle of a smooth, compact Riemann manifold $\mathrm{M}$. The geodesic flow $G: \mathbf{R} \times S M \rightarrow S M$ is defined by

$$
G(t,(x, v))=G_{t}(x, v)=\left(\gamma_{v}(t), \dot{\gamma}_{v}(t)\right),
$$

where $\gamma_{v}$ is the unique geodesic satisfying $\gamma_{v}(0)=x$ and $\dot{\gamma}_{v}(0)=v$.

A measure $\mu$ on $S M$ is invariant under the geodesic flow, if $G_{t *} \mu=\mu$ for every $t \in \mathbf{R}$, that is, $\mu\left(G_{t}^{-1}(A)\right)=\mu(A)$ for all subsets $A \subset S M$.

The main result of this section is the following theorem.

Theorem 4.2. Let $M$ be a smooth, compact Riemann surface, let $\mu$ be a Borel regular probability measure on the unit tangent bundle $S M$, and let $\Pi: S M \rightarrow M$ be the natural projection. If $\mu$ is invariant under the geodesic flow, then for $1<q \leq 2$

$$
\underline{D}_{q}\left(\Pi_{*} \mu\right)=\underline{D}_{q}^{2}(\mu) \quad \text { and } \quad \bar{D}_{q}\left(\Pi_{*} \mu\right)=\bar{D}_{q}^{2}(\mu)
$$

For the proof of Theorem 4.2 we need to prove a few technical lemmas, but first we are going to fix our notation, which is similar to that of Section 3 in [JJLe]. The invariance of the measure $\mu$ and the compactness of the manifold $M$ imply that there exist sets $U_{1}, \ldots, U_{k}$ which cover $S M$ and for the restricted measures $\mu_{j}=\left.\mu\right|_{U_{j}}$ the following holds: $\mu_{j}=\psi_{*}\left(\nu_{j} \times \mathscr{L}^{1}\right)$ is the image measure under a bi-Lipschitz mapping $\psi$ from a compact set $\tilde{I}=[0,1]^{3} \subset \mathbf{R}^{3}$ to its image $\psi(\tilde{I}) \subset S M$, and $\nu_{j}$ is a finite measure on $[0,1]^{2}$ for every $j \in\{1, \ldots, k\}$. This kind of measures $\mu_{j}$ are from now on called locally invariant. For more details see Section 3 in [JJLe].

It holds that

$$
\underline{D}_{q}\left(\Pi_{*} \mu\right)=\min _{j} \underline{D}_{q}\left(\Pi_{*} \mu_{j}\right) \quad \text { and } \quad \bar{D}_{q}\left(\Pi_{*} \mu\right)=\min _{j} \bar{D}_{q}\left(\Pi_{*} \mu_{j}\right) .
$$

This can be seen by covering the manifold $M$ by finitely many coordinate neighbourhoods and considering the measures in local coordinates. In local coordinates (4.1) is easy to prove using the definitions (2.1) and (2.2) for the $q$-dimensions and applying Hölder's inequality.

By (4.1) it is enough to prove Theorem 4.2 for the measures $\mu_{j}$. To do this, we take some $j \in\{1, \ldots, k\}$ and denote $\tilde{\mu}=\mu_{j}$ and $\nu=\nu_{j}$. In [JJLe] it was shown that there exists a transversal mapping $P:[0,1] \times[0,1]^{2} \rightarrow \mathbf{R}, P(t, x)=P_{t}(x)$ such that the dimension of $(\Phi \circ \Pi)_{*} \tilde{\mu}$ is the same than that of the measure $\mu^{\prime}$ defined by

$$
\int_{\mathbf{R}^{2}} g(x, t) d \mu^{\prime}(x, t)=\int_{0}^{1} \int_{\mathbf{R}} g(x, t) d P_{t *} \nu(x) d \mathscr{L}^{1}(t)
$$

for all Borel functions $g: \mathbf{R} \times[0,1] \rightarrow[0, \infty]$. Here $\Phi$ is a coordinate mapping defined on an open set $U \subset M$ for which $[0,1]^{2} \subset \Phi(U)$. For details see Sections 3 and 4 in [JJLe].

In order to make use of the condition (4.2), we prove a lemma which gives a lower bound for the $q$-dimension of the measure $\Pi_{*} \tilde{\mu}$.

Lemma 4.3. Let $1<q \leq 2$ and let $\mu^{\prime}$ be the measure defined by equation (4.2). Then

$$
D_{q}^{2}\left((\Phi \circ \Pi)_{*} \tilde{\mu}\right)=D_{q}^{2}\left(\mu^{\prime}\right) \geq D_{q}^{1}(\nu)+1 .
$$

Here $D_{q}^{m}(\cdot)$ is either the lower or the upper modified q-dimension. 
Proof. Let $0<p \leq 1$. Then by the definition of $\mu^{\prime}$ and equation (2.3) it holds that

$$
\begin{aligned}
& \int F_{\mu^{\prime}}^{2}((x, t), r)^{p} d \mu^{\prime}(x, t)=\int\left(2 r^{2} \int_{r}^{\infty} h^{-3} \mu^{\prime}(B((x, t), h)) d h\right)^{p} d \mu^{\prime}(x, t) \\
& \leq \int_{0}^{1} \int_{\mathbf{R}}\left(2 r^{2} \int_{r}^{\infty} h^{-3} \int_{t-h}^{t+h} P_{s *} \nu(B(x, h)) d \mathscr{L}^{1}(s) d h\right)^{p} d P_{t *} \nu(x) d \mathscr{L}^{1}(t) \\
& =\int_{0}^{1} \int_{[0,1]^{2}}\left(2 r^{2} \int_{r}^{\infty} h^{-3} \int_{t-h}^{t+h} \nu\left(P_{s}^{-1}\left(B\left(P_{t}(x), h\right)\right)\right) d \mathscr{L}^{1}(s) d h\right)^{p} d \nu(x) d \mathscr{L}^{1}(t) .
\end{aligned}
$$

Let $y \in P_{s}^{-1}\left(B\left(P_{t}(x), h\right)\right)$. Then by inequality (3.1)

$$
h>\left|P_{t}(x)-P_{s}(y)\right| \geq\left|P_{t}(x)-P_{t}(y)\right|-\left|P_{t}(y)-P_{s}(y)\right| \geq\left|P_{t}(x)-P_{t}(y)\right|-C_{1} h,
$$

which implies $\left|P_{t}(x)-P_{t}(y)\right|<\left(C_{1}+1\right) h$. Thus $y \in P_{t}^{-1}\left(B\left(P_{t}(x), C h\right)\right)$ for $C=C_{1}+1$. It follows that

$$
\begin{aligned}
& \int F_{\mu^{\prime}}^{2}((x, t), r)^{p} d \mu^{\prime}(x, t) \\
& \leq \int_{0}^{1} \int_{[0,1]^{2}}\left(2 r^{2} \int_{r}^{\infty} h^{-3} \int_{t-h}^{t+h} \nu\left(P_{t}^{-1}\left(B\left(P_{t}(x), C h\right)\right) d \mathscr{L}^{1}(s) d h\right)^{p} d \nu(x) d \mathscr{L}^{1}(t)\right. \\
& =\int_{0}^{1} \int_{\mathbf{R}}\left(4 r^{2} \int_{r}^{\infty} h^{-2} P_{t *} \nu((B(x, C h))) d h\right)^{p} d P_{t *} \nu(x) d \mathscr{L}^{1}(t) \\
& =C^{\prime} r^{p} \int_{0}^{1} \int_{\mathbf{R}} F_{P_{t *} \nu}^{1}(x, C r)^{p} d P_{t *} \nu(x) d \mathscr{L}^{1}(t) .
\end{aligned}
$$

Let $\epsilon>0$. First using [FO, Proposition 2.8] and then covering the interval $[0,1]$ by a finite number of balls $B\left(t_{i}, r_{i}\right)$ given by Proposition 3.3, we have by inequality (3.6) that

$$
\begin{aligned}
\int F_{\mu^{\prime}}^{2}((x, t), r)^{p} d \mu^{\prime}(x, t) & \leq C^{\prime} r^{p-\epsilon} \int_{0}^{1} \int_{\mathbf{R}} P_{t *} \nu(B(x, C r))^{p} d P_{t *} \nu(x) d \mathscr{L}^{1}(t) \\
& \leq C^{\prime} r^{p-\epsilon} \sum_{i=1}^{k} \int_{B\left(t_{i}, r_{i}\right)} \int_{\mathbf{R}} P_{t *} \nu(B(x, C r))^{p} d P_{t *} \nu(x) d \mathscr{L}^{1}(t) \\
& \leq \widetilde{C} r^{p-\epsilon} \int_{[0,1]^{2}} F_{\nu}^{1}(x, C r)^{p} d \nu(x)
\end{aligned}
$$

for all small enough $\mathrm{r}$. Using this for $p=q-1$, we have that

$$
D_{q}^{2}\left(\mu^{\prime}\right) \geq 1-\frac{\epsilon}{q-1}+D_{q}^{1}(\nu)
$$

Since $\epsilon>0$ was arbitrary, the claim is proved.

Remark 4.4. Note that in the previous lemma it is crucial that the measure $\mu^{\prime}$ is defined by using the images of $\nu$ under a transversal mapping $P$. If for all $t \in[0,1]$ we have a compactly supported Borel regular measure $\nu_{t}$ on $\mathbf{R}$, if $\mu^{\prime}$ is a Borel regular measure on $\mathbf{R} \times[0,1]$ defined by

$$
\int_{\mathbf{R}^{2}} g(x, t) d \mu^{\prime}(x, t)=\int_{0}^{1} \int_{\mathbf{R}} g(x, t) d \nu_{t}(x) d \mathscr{L}^{1}(t),
$$


and if there exists $\alpha>0$ such that $D_{q}^{1}\left(\nu_{t}\right) \geq \alpha$ for $\mathscr{L}^{1}$-almost all $t \in[0,1]$, then it does not in general hold that $D_{q}^{2}\left(\mu^{\prime}\right) \geq \alpha+1$. For the Hausdorff dimension and the packing dimension the above inequality holds. The difference follows from the fact that the $q$-dimensions of slices of a measure do not behave as well as the Hausdorff or the packing dimension. For the definition of these slices see [Mat3, Chapter 10]. For the Hausdorff and the packing dimension we have the following: Let $\vartheta$ be a compactly supported Borel regular measure on $\mathbf{R}^{2}$ such that $\operatorname{dim}(\vartheta)>1$ and $\left.P_{V *} \vartheta \ll \mathscr{H}^{1}\right|_{V}$ for all lines $V \in G(2,1)$, where $P_{V}$ is the orthogonal projection onto the line $V$. Then for all $V \in G(2,1)$ and almost all $t \in V^{\perp}$,

$$
\mathscr{H}^{1} \text {-essinf }\left\{\operatorname{dim}\left(\vartheta_{V, t}\right): \vartheta_{V, t} \neq 0\right\} \leq \operatorname{dim}(\vartheta)-1 .
$$

(See the proof of Lemma 3.1 in [JM].) For the $q$-dimensions this does not hold, as the following example shows. Let $1<q \leq 2$ and let $(2-q) / q<\lambda<1$. Define a measure $\vartheta$ by $d \vartheta=\left.\|x\|^{-(\lambda+1)} d \mathscr{L}^{2}\right|_{[0,1] \times[0,1]}$, and let $V$ be the $x$-axis. Then for the slices $\vartheta_{V, t}, t \in[0,1]$, it holds that

$$
D_{q}\left(\vartheta_{V, t}\right)=D_{q}^{1}\left(\vartheta_{V, t}\right)=1 \text { whenever } t \neq 0,
$$

but for the measure $\vartheta$ we have

$$
D_{q}(\vartheta)=D_{q}^{2}(\vartheta)=\frac{q(1-\lambda)}{q-1}<2 .
$$

This explains why we cannot use similar methods as in [JJLe] and [Le] to prove the dimension bound for the measure defined by equation (4.2). This also gives an answer to the question that arises naturally from [FO]. In Proposition 4.2 they consider the $q$-dimension of slices of a measure for $0<q<1$ and remark that similar methods cannot be used for $q>1$. The above example shows that for $1<q \leq 2$ the proposition does not hold.

Lemma 4.5. For $1<q \leq 2$ it holds that

$$
D_{q}\left((\Phi \circ \Pi)_{*} \tilde{\mu}\right)=D_{q}^{2}(\tilde{\mu}),
$$

where $D_{q}(\cdot)$ and $D_{q}^{2}(\cdot)$ are either the lower or the upper q-dimensions.

Proof. First we will show that

$$
D_{q}^{2}(\tilde{\mu})=D_{q}^{1}(\nu)+1 .
$$

Since $\psi$ is a bi-Lipschitz mapping, we have that for all $(y, s) \in \tilde{I}$ and $r>0$

$$
\begin{aligned}
F_{\nu}^{1}(y, r) & =r \int_{r}^{\infty} \frac{\nu(B(y, h))}{h^{2}} d h=r \int_{r}^{\infty} \frac{\nu(B(y, h)) \cdot h}{h^{3}} d h \\
& \asymp r \int_{r}^{\infty} \frac{\left(\nu \times \mathscr{L}^{1}\right)(B((y, s), h))}{h^{3}} d h \\
& \asymp r \int_{r}^{\infty} \frac{\psi_{*}\left(\nu \times \mathscr{L}^{1}\right)(B(\psi(y, s), h))}{h^{3}} d h \\
& \asymp \frac{F_{\psi_{*}\left(\nu \times \mathscr{L}^{1}\right)}^{2}(\psi(y, s), r)}{r}=\frac{F_{\tilde{\mu}}^{2}(\psi(y, s), r)}{r} .
\end{aligned}
$$

By $A \asymp B$ we denote that the ratio $\frac{A}{B}$ is bounded away from 0 and $\infty$ by some constants. Above these constants are independent of $y, s$ and $r$. By raising both sides 
of the equation (4.5) to the power $q-1$ and integrating with respect to $\nu \times\left.\mathscr{L}^{1}\right|_{[0,1]}$ we see that

$$
\int F_{\nu}^{1}(y, r)^{q-1} d \nu(y) \asymp r^{-(q-1)} \int F_{\tilde{\mu}}^{2}(x, r)^{q-1} d \tilde{\mu}(x) .
$$

Equality (4.4) follows directly from this and the definition of $D_{q}^{m}(\cdot)$.

By Proposition 3.5 we have that $D_{q}\left((\Phi \circ \Pi)_{*} \tilde{\mu}\right)=D_{q}^{2}\left((\Phi \circ \Pi)_{*} \tilde{\mu}\right)$. Thus by Lemma 4.3 and equality (4.4)

$$
D_{q}\left((\Phi \circ \Pi)_{*} \tilde{\mu}\right) \geq D_{q}^{1}(\nu)+1=D_{q}^{2}(\tilde{\mu}) .
$$

On the other hand, the fact that the dimension $D_{q}^{m}(\cdot)$ does not increase under Lipschitz mappings implies that

$$
D_{q}\left((\Phi \circ \Pi)_{*} \tilde{\mu}\right)=D_{q}^{2}\left((\Phi \circ \Pi)_{*} \tilde{\mu}\right) \leq D_{q}^{2}(\tilde{\mu}),
$$

proving the claim.

Proof of Theorem 4.2. The claim follows directly from Lemma 4.5 and the fact that $\Phi$ does not change the dimension as a bi-Lipschitz mapping.

Example 4.6. Proposition 3.2 and Theorem 4.2 imply that as in the case of the Hausdorff dimension, the lower $q$-dimension is preserved under the natural projection in the two-dimensional case. However, the upper $q$-dimension may decrease under the projection. Here we will give an example of such a measure. We use the measure constructed in [FM, Example 5.1] to obtain a locally invariant measure whose upper $q$-dimension for $1<q \leq 2$ decreases under the natural projection. Using their example we obtain for $0<\underline{d}<\bar{d}<2, \underline{d}<1$ a finite, compactly supported measure $\nu$ on $[0,1]^{2}$, for which

Defining

$$
\bar{D}_{q}^{1}(\nu) \leq \frac{\bar{d}\left(1-\frac{1}{2} \underline{d}\right)}{1+\frac{1}{2} \bar{d}-\underline{d}}<\bar{D}_{q}(\nu) .
$$

$$
\tilde{\mu}=\psi_{*}\left(\nu \times \mathscr{L}^{1}\right)
$$

we obtain a locally invariant measure for which

$$
\bar{D}_{q}\left(\Pi_{*} \tilde{\mu}\right)=\bar{D}_{q}^{2}(\tilde{\mu})=\bar{D}_{q}^{1}(\nu)+1<\bar{D}_{q}(\nu)+1=\bar{D}_{q}(\tilde{\mu}) .
$$

\section{References}

[FH] FAlconer, K. J., and J. D. Howroyd: Packing dimensions of projections and dimension profiles. - Math. Proc. Cambridge Philos. Soc. 121, 1997, 269-286.

[FM] Falconer, K. J., and P. Mattila: The packing dimensions of projections and sections of measures. - Math. Proc. Cambridge Philos. Soc. 119, 1996, 695-713.

[FO] Falconer, K. J., and T. C. O'Neil: Convolutions and the geometry of multifractal measures. - Math. Nachr. 204, 1999, 61-82.

[HP] Hentschel, H., and I. Procaccia: The infinite number of generalized dimensions of fractals and strange attractors. - Physica D 8, 1983, 435-444.

[HT] Hu, X., and J. TAYLOR: Fractal properties of products and projections of measures in $\mathbf{R}^{n}$. - Math. Proc. Cambridge Philos. Soc. 115, 1994, 527-544.

[HK] Hunt, B. R., and V.Y. KAloshin: How projections affect the dimension spectrum of fractal measures. - Nonlinearity 10, 1997, 1031-1046.

[JJ] JÄRVENPÄÄ, E., and M. JÄRVENPÄÄ: Linear mappings and generalized upper spectrum for dimensions. - Nonlinearity 12, 1999, 475-493. 
[JJLe] JÄrvenpäÄ, E., M. JÄrvenPÄÄ, and M. LeikAS: (Non)regularity of projections of measures invariant under geodesic flow. - Comm. Math. Phys. 254, 2005, 695-717.

[JJL] JÄrVEnpäÄ, E., M. JÄrVEnpÄÄ, and M. Llorente: Local dimensions of sliced measures and stability of packing dimensions of sections of sets. - Adv. Math. 183, 2004, 127-154.

[JJN] JÄrvenpäÄ, E., M. JÄrvenPÄÄ, and J. NIEMELÄ: Transversal mappings between manifolds and non-trivial measures on visible parts. - Real Anal. Exchange (2) 30, 2004, 675-688.

[JM] JÄrVEnPä̈̈, M., and P. Mattila: Hausdorff and packing dimensions and sections of measures. - Mathematika 45, 1998, 55-77.

[K] Kaufman, R.: On Hausdorff dimension of projections. - Mathematika 15, 1968, 153-155.

[LL] Ledrappier, F., and E. Lindenstrauss: On the projections of measures invariant under the geodesic flow. - Int. Math. Res. Not. 9, 2003, 511-526.

[Le] Leikas, M.: Packing dimensions, transversal mappings and geodesic flows. - Ann. Acad. Sci. Fenn. Math. 29, 2004, 489-500.

[Mar] Marstrand, M.: Some fundamental geometrical properties of plane sets of fractional dimension. - Proc. London Math. Soc. (3) 4, 1954, 257-302.

[Mat1] MatTila, P.: Hausdorff dimension, orthogonal projections and intersections with planes. Ann. Acad. Sci. Fenn. Math. 1, 1975, 227-244.

[Mat2] Matrila, P.: Orthogonal projections, Riesz capacities and Minkowski content. - Indiana Univ. Math. J. 39, 1990, 185-198.

[Mat3] Mattila, P.: Geometry of sets and measures in Euclidean spaces. - Cambridge Univ. Press, 1995.

[PS] Peres, Y., and W. Schlag: Smoothness of projections, Bernoulli convolutions and the dimension of exceptions. - Duke Math. J. (2) 102, 2000, 193-251.

Received 9 November 2009 